\title{
Erratum to: Symplectic Dolbeault operators on Kähler manifolds
}

\author{
Eric O. Korman
}

Published online: 2 June 2013

C) Springer Science+Business Media Dordrecht 2013

\section{Erratum to: Ann Glob Anal Geom DOI 10.1007/s10455-013-9369-x}

The Hermitian structure used for a flag manifold $G / T$ in Sect. 4 is not a Kähler structure unless $G$ is a product of $S U(2)$ 's. This is because the connection used is the canonical connection on the homogeneous space $G / T$, which has non-zero torsion since $\left[\mathfrak{t}^{\perp}, \mathfrak{t}^{\perp}\right] \nsubseteq \mathfrak{t}$. Indeed, using the metric to lower indices, the torsion at $e T$ is the 3 -form given by

$$
(X, Y, Z) \mapsto-g_{0}([X, Y], Z), \quad X, Y, Z \in \mathfrak{t}^{\perp} \simeq T_{e T} G / T \quad[1] .
$$

Such a structure on $G / T$ turns it into what is called a "Kähler with torsion (KT)" manifold. The general results and constructions established in the previous sections carry over unchanged to KT manifolds except for Proposition 3 which states that the symplectic Dirac operators $D$ and $\tilde{D}$ are formally self-adjoint. For connections with torsion and parallel complex structure, a sufficient condition for $D$ and $\tilde{D}$ to be self-adjoint is the vanishing of the torsion vector field, defined by

$$
\mathcal{T}=\sum_{j=1}^{n} \mathbf{T}\left(a_{j}, b_{j}\right),
$$

where $\mathbf{T}$ is the torsion of $\nabla$ and $\left\{a_{1}, \ldots, a_{n}, b_{1}, \ldots, b_{n}\right\}$ is a symplectic frame [2]. In the case of flag manifolds, a symplectic basis at $e T$ is proportional to $\left\{Z_{\alpha}, Z_{-\alpha}\right\}$ where $Z_{\alpha}$ is a root vector for $\alpha$. Since $\mathbf{T}\left(Z_{\alpha}, Z_{-\alpha}\right)=-\left[Z_{\alpha}, Z_{-\alpha}\right]_{\mathfrak{t}^{\perp}}=0$, we see that $\mathcal{T}$ vanishes. Thus the results concerning flag manifolds are correct using the symplectic Dolbeault operators corresponding to their KT structures.

The online version of the original article can be found under doi:10.1007/s10455-013-9369-x.

E. O. Korman $(\varangle)$

Department of Mathematics, University of Pennsylvania, Philadelphia, PA 19104, USA

e-mail: ekorman@math.upenn.edu 


\section{References}

1. Kobayashi, S., Nomizum, K.: Foundations of Differential Geometry, vol. 2, pp. 192-193. Interscience Publishers, New York (1969)

2. Habermann, K., Habermann, L.: Introduction to symplectic Dirac operators. In: Lecture Notes in Mathematics. Springer, Berlin (2006) 